\title{
MOLECULAR EVENTS IN THE ASSEMBLY OF RETROVIRUS PARTICLES
}

\author{
Michael Sakalian and Eric Hunter \\ University of Alabama at Birmingham \\ Department of Microbiology and The UAB AIDS Center \\ UAB Station \\ Birmingham, Alabama 35294
}

\section{ABSTRACT}

Retrovirus assembly results from the ability of a single gene product, the gag polyprotein precursor, to coalesce into a spherical particle capable of release from the cell. In conjunction with this primary process of capsid formation additional viral gene products such as the replicative enzymes and envelope glycoproteins as well as the genomic RNA are incorporated to form an infectious virus.

\section{INTRODUCTION}

Retrovirus assembly involves a process in which a large number of chemically distinct macromolecules migrate through different transport pathways to the plasma membrane of the cell (Figure 1) where they are assembled into a nascent viral particle. The capsid of the virus is assembled from a large number of polyprotein precursors that must be transported through the cytoplasm to the underside of the plasma membrane. The membrane-spanning viral glycoproteins, on the other hand, must be transported through the secretory pathway of the cell to the cell surface where they co-localize with the nascent, membrane-extruding capsid. At a point still undetermined in the capsid assembly process, genome-length viral RNA molecules, along with necessary cell-derived tRNAs, must become associated with both capsid and polymerase components. By understanding the molecular basis of the interaction involved in assembly of the pathogenic human retroviruses, it may be possible to develop methods to therapeutically intervene in their replication. 


\section{CAPSID ASSEMBLY}

\subsection{The gag Gene Product Directs Virus Assembly}

All replication competent retroviruses contain four genes that encode the structural and enzymatic components of the virion (Figure 2). These are gag (capsid protein), pro (aspartyl proteinase), pol (reverse transcriptase and integrase enzymes) and env (envelope glycoprotein). Unlike most other enveloped RNA viruses in which the viral glycoproteins appear to catalyze virus particle formation, assembly and release of retrovirus particles occurs when capsid proteins are produced in the absence of the other gene products. Thus the product of this gene has the necessary structural information to mediate intracellular transport, to direct self-assembly into the capsid shell and to catalyze the process of membrane extrusion known as budding (Craven and Parent, 1996; Kräusslich and Welker, 1996; Hunter, 1994).

The gag gene product, a polyprotein precursor, is translated on free polyribosomes from an unspliced, genome length mRNA (Eisenman et al., 1974). In most retroviruses, the nascent Gag polyproteins are transported directly to the plasma membrane where as-

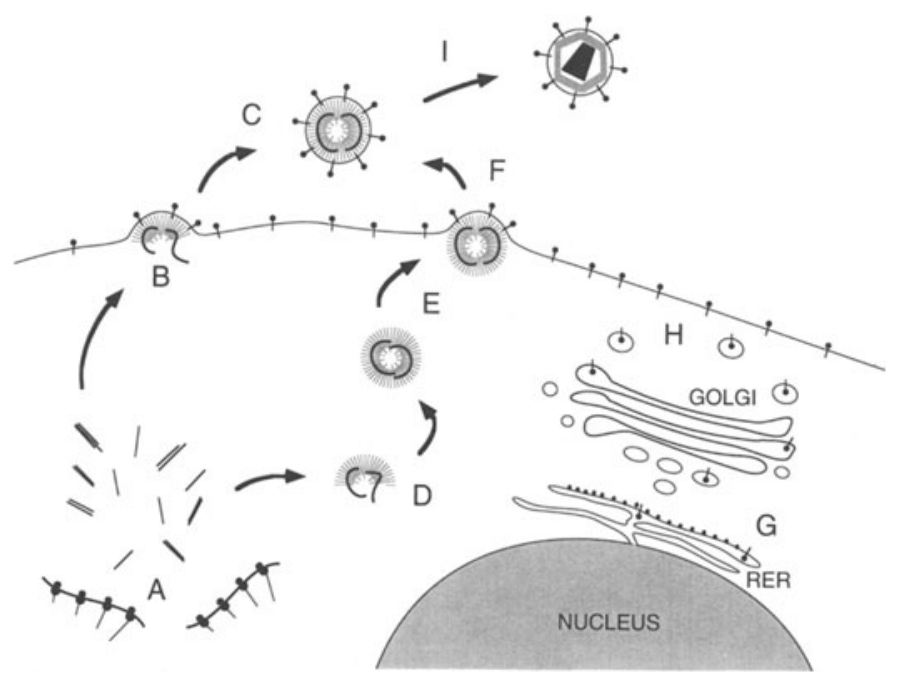

Figure 1. Schematic representation of retrovirus assembly. The morphogenesis of both C-type (A to B) and B/Dtype (A to D-F) retroviruses is depicted. The Gag and Gag-Pol precursors are synthesized on free polyribosomes (A). In the case of C-type morphogenesis (i.e. HIV), the capsid precursors are transported, either individually or in small aggregates, directly to the plasma membrane where capsid assembly and budding (B) occur simultaneously. In contrast, for the B/D-type viruses, the capsid precursors are first transported to an intracytoplasmic assembly site (D). Immature capsids are then transported (E) to the plasma membrane where they induce budding to occur (F). The envelope glycoproteins of these viruses are translated on membrane bound polyribosomes and then traverse a common route to the plasma membrane (G-H), the secretory pathway of the cell. During the budding process, the viral glycoproteins presumably migrate to the same site in the membrane and are incorporated into the virus. For both morphogenetic types of retroviruses, an immature virus particle is released from the cell (C) and rapidly undergoes maturation (I). The mature virus particles have dense cores with a variable morphology characteristic of individual virus species. 
sembly of the capsid shell and membrane extrusion occur simultaneously (Figure 1). Viruses that undergo this form of morphogenesis are known as type- $\mathrm{C}$ viruses and include the avian and mammalian leukemia/sarcoma viruses (e.g. Rous sarcoma, avian leukosis, and murine leukemia virus). The pathogenic human viruses, human T-cell leukemia virus and human immunodeficiency virus (HTLV-I and HIV), assemble their capsids in a similar fashion. In the second morphogenic class of viruses, the Gag precursors appear to be targeted to an intracytoplasmic site where capsid assembly occurs. These preassembled immature (A-type) particles are then transported to the plasma membrane where they undergo budding and envelopment (Figure 1). Viruses that undergo this process of assembly and release include the type-B, mouse mammary tumor virus (MMTV), the type-D, Mason-Pfizer monkey virus (M-PMV) and related simian retroviruses (SRV.1-5) as well as members of the spumavirus family. Despite the different morphogenic pathways, the process by which Gag precursors self-assemble into capsids is probably similar for the type- $\mathrm{C}$ and type-B/D viruses since a single amino acid change within the gag gene product of M-PMV was shown to divert it to the type-C morphogenic pathway (Hunter, 1994). Irrespective of the pathway to virus release, the newly budded-off virions have a common immature morphology. In thin-section electron microscopy the capsid shell appears as an electron opaque band in tight apposition to the membrane with an electron lucent center. During the process of virus maturation, the capsid polyprotein precursors are cleaved by the virus-encoded aspartyl proteinase, and this structure collapses into an electron dense core with a morphology characteristic of the virus family. (Reviewed in Nermut and Hockley, 1996; Gelderblom, 1991.)

\subsection{Organization and Functions of the gag Gene Products in Virion Particles}

The Gag polyprotein precursor functions as the primary building block in virus capsid assembly and during maturation it is cleaved by the viral proteinase to yield a number of individual proteins that make up the mature virion (Figure 2B). The translation of these products as a precursor protein thus ensures that equimolar amounts of each of the struc-

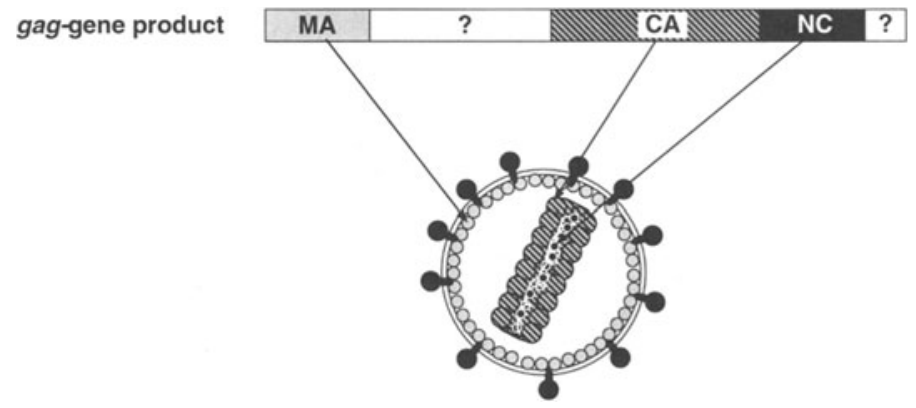

Figure 2. Organization of a generic retrovirus gag gene product. The Gag polyprotein precursor consists of the conserved matrix (MA), capsid (CA) and nucleocapsid (NC) protein domains. Unshaded boxes represent regions for which no common defined function has been established. Cleavage by the viral protease results in production of the matured Gag proteins the locations of which are shown in the schematic representation of a retrovirus particle. 
tural proteins are incorporated into the virus. While the size and protein content of the precursor varies between different retroviral families, at least three gag-encoded proteins are found in all retroviruses; these are the matrix protein (MA), the capsid protein (CA) and the nucleocapsid protein (NC) (Figure 2) (Nermut and Hockley, 1996; Leis et al., 1988). The matrix protein is closely associated with the viral membrane. In most retroviruses, MA is modified co-translationally by the $\mathrm{N}$-terminal addition of a myristic acid residue which is critical for its function. The CA protein forms the major protein component of the electron dense core in mature virions, where it appears to form a protein shell into which the virion RNA genome and replicative enzymes are packed (Gelderblom, 1991). The NC protein is located within the CA-derived shell (Figure 2C) where it is found associated with the viral RNA genome (Linial and Miller, 1990). This domain of the Gag precursor, in all retroviruses but the spumaviridae, contains a conserved cysteine-histidine rich, zincfinger-like, region (Cys-X2-Cys-X4-His-X4-Cys) that is thought to play an important role in the specific packaging of viral RNA into the assembling virus (Katz and Jentoft, 1989).

The arrangement of the proteins on the precursor $\left(\mathrm{NH}_{2}-\mathrm{MA}-\mathrm{CA}-\mathrm{NC}-\mathrm{COOH}\right)$ reflects their position in both the immature capsid and the matured virion, where they appear to form concentric shells of protein after cleavage from the precursor (reviewed in Hunter, 1994). Despite a common organization, Gag precursors from different retroviruses share little amino acid sequence homology. Functional homologies must thus be reflected at the level of three-dimensional structure, as has been observed between retroviral proteinases (Weber, 1990) and among the MA protein structures of HIV, SIV, BLV (Matthews et al., 1996) and M-PMV (Conte et al., 1997) which maintain very similar three-dimensional conformations.

In addition to the functionally conserved MA, CA, and NC proteins, the Gag precursor can, depending on the virus encoding it, contain additional peptide sequences (Figure 2) whose function in virus assembly and future cycles of infection is for the most part obscure. Several of the undesignated peptide domains are short and may represent spacer peptides that allow the correct folding of the precursor protein for assembly and subsequent processing into the mature virion proteins. Alternately, since the precursor protein is the assembly unit for capsid construction, such regions could represent novel domains of the precursor that function transiently during the process of virus assembly and which are then dispensed with during the process of virus maturation.

\subsection{Biosynthesis and Assembly of pro and pol Gene Products}

The viral proteinase and reverse-transcriptase/integrase enzymes pose a translational problem for most retroviruses since their coding sequences are located in the middle of the genome and they also must be targeted intracellularly for assembly into virions. These problems are solved in most retroviruses by translating the enzymes from an unspliced, genome-length mRNA as long polyprotein precursors that represent $\mathrm{C}$-terminal extensions of the gag gene product itself. The Gag sequence present at the amino-terminus of the Pro and Pol precursors is thought to target the molecules to the site of virus assembly and to facilitate their inclusion into the nascent virion. In many retroviruses synthesis of these Gag-Pro-Pol fusion proteins is achieved by way of a ribosomal frameshift mechanism. This process which occurs with an efficiency of approximately $5 \%$ also provides a mechanism for the virus to regulate the level of enzymatic proteins incorporated into virions. For some viruses, such as M-PMV and HTLV, pro lies within its own reading frame between gag and pol. Two extended Gag precursors, Gag-Pro, and Gag-Pro-Pol, are therefore produced as the result of one or two frameshift events respectively (Jacks, 1990). 
MuLV, utilizes a subtly different mechanism and expresses pro and pol through the use of a suppressor tRNA which allows ribosomes to read past the stop codon. Foamy viruses, exemplified by the human spumaretrovirus (HSRV), are unique in that they express their pro and pol genes by translation of a separate spliced message (Bodem et al., 1996). Since the human spumavirus does not express pro and pol as a fusion with gag it is unclear how these enzymes are incorporated into virus particles.

\subsection{Transport of Capsid Proteins to the Site of Assembly}

The mechanism(s) by which the capsid precursor proteins are directed to the site of assembly remains for the most part unknown but is primarily mediated by the MA domain of the Gag precursor (Kräusslich and Welker, 1996). In the C-type retroviruses, the Gag and Gag-Pol precursors are transported directly to the plasma membrane where self-assembly into capsids occurs. In B- and D-type retroviruses, where capsid assembly and virus budding are discrete events, transport involves two stages: in the first capsid precursors are directed to an intracytoplasmic assembly site; in the second preassembled capsids are transported to the plasma membrane (Hunter, 1994). A recent genetic dissection of this process suggests that Gag-containing precursors of D-type viruses express a dominant sorting signal that targets the proteins to the initial assembly site (Figure 1). A point mutation within the MA coding domain of M-PMV abrogated this targeting and resulted in the direct, type-C-like, transport of precursors to the plasma membrane where efficient capsid assembly occurred. This result demonstrates that while the morphogenic pathway of a retrovirus can be altered the assembly of particles utilizes a common mechanism.

Transport of the preassembled capsids of D-type viruses to the plasma membrane appears to be dependent on an active process rather than passive diffusion since a variety of mutations within the MA domain of M-PMV result in the accumulation of discrete clusters of immature capsids deep within the cytoplasm-apparently unable to exit the site of assembly. This phenotype was initially observed for mutations that blocked the co-translational addition of myristic acid to the amino-terminus of the Gag precursors. However, two additional transport-defective M-PMV mutants with single amino acid changes in MA have similar phenotypes even though their Gag precursors are myristylated normally.

In the C-type retroviruses, the MA domain of the Gag precursor also plays a major role in targeting capsid components to the plasma membrane site of assembly. Mutations which block myristylation interfere with this process (reviewed in Kräusslich and Welker, 1996; Hunter, 1994). Mutations in MA can redirect Gag precursors to a new site for assembly, as with the morphogenesis mutant of M-PMV described above. Similarly, a deletion within the MA protein of HIV-1 results in the targeting of capsid precursors to the endoplasmic reticulum where they assemble and bud into the lumen of this compartment (Kräusslich and Welker, 1996).

By what pathway(s) might preassembled capsids of the B- and D-type viruses or the Gag-containing precursors of the C-type viruses be transported to the plasma membrane? Several studies have suggested that cytoskeletal elements might be involved in this process. MuLV, HIV, and HSRV Gag have been shown to be associated with cytoskeletal elements. Moreover, electron micrographs of MMTV- and RSV-infected cells have shown microfilaments in close association with budding viruses (reviewed in Kräusslich and Welker, 1996). Recent experiments indicate that the process is an active rather than a passive one since it appears to require energy. In cells treated with agents that block ATP synthesis M-PMV capsids are unable to migrate to the plasma membrane from sites deep 
within the cytoplasm (Weldon and Hunter, 1997). A requirement for energy suggests a possible role for chaperonins in the transport process.

\subsection{Assembly of the Capsid Shell}

Whether the Gag precursor proteins are first transported to an intracytoplasmic site or to the plasma membrane they must associate with other precursors in a regular and symmetrical fashion to self-assemble into the nascent capsid. Mutational analyses of gag genes have attempted to define what portions of the Gag precursor are required for particle formation itself and which domains might have other functions in the virus replication cycle. It is important to recognize that assembly domains within the Gag precursor may not necessarily reside within the boundaries of the mature cleavage products of Gag but may span cleavage sites and thus would be destroyed during the process of virus maturation. In this way the process of maturation would define the transition from an assembly function for Gag to an entry/infection function where there is a requirement for efficient disassembly and release of a transcriptionally active core upon infection of a new cell.

Deletion analysis has identified three essential assembly domains designated $\mathrm{M}, \mathrm{L}$, and I to reflect their specific functions for Membrane association, Late budding release, and Gag-Gag Interaction (Craven and Parent, 1996). Assembly domain M lies at the N-terminus of Gag and appears to be necessary for membrane association. Domain L has been identified for both RSV and M-PMV to contain a PPPY motif that is required for final release of budding particles. In RSV this sequence is located in the $\mathrm{p} 2 \mathrm{~b}$ spacer peptide between MA and p10. In M-PMV the same sequence is found within the pp16 region. Mutations in this sequence of either virus result in budding particles that remain attached to the plasma membrane (Yasuda and Hunter, 1997; Craven and Parent, 1996). In HIV the equivalent role is played by the carboxy-terminal p6 domain. Chimeric Gag proteins containing $\mathrm{L}$ domains from HIV or RSV in either the $\mathrm{p} 2 \mathrm{~b}$ or carboxy-terminal position have been shown to assemble and bud normally supporting the idea that the mechanisms by which retroviruses assemble are fundamentally equivalent.

The Gag-Gag interaction or I domain is essential for the production of particles with the correct density and size. For RSV, the I domain has been narrowed down to the carboxy-terminal end of the CA domain and half of the NC domain (Craven and Parent, 1996). The Gag proteins of HIV and MuLV also require equivalent regions for the production of particles with the correct density. This domain could establish the correct proteinprotein interactions which allow the tight packing of Gag molecules during assembly or since this region is implicated in RNA packaging, it may influence particle density by directly mediating RNA encapsidation. Thus RNA could serve as a necessary scaffold upon which Gag proteins tightly pack during particle assembly — an idea supported by recent in vitro experiments (see below).

In some retroviruses, additional domains of Gag or other gene products may influence the efficiency of particle assembly without being essential to the process itself. For example, in M-PMV the p12 coding region can be deleted completely without affecting capsid assembly if Gag precursors are expressed at high levels. In contrast, such mutant Gag precursors are defective in assembly when expressed at levels typical of a normal virus infection (Sommerfelt et al., 1992).

How might Gag precursors become organized into a virus particle? Some researchers have hypothesized that Gag precursors pack together in a regular fashion to form a fullerine-like structure similar to those of icosahedral viruses (Nermut and Hockley, 1996). However, recent cryoelectron microscopy studies have demonstrated a lack of 
icosahedral symmetry as well as a large heterogeneity in particle size raising questions as to the organization of $\mathrm{Gag}$ molecules in capsids.

\subsection{Assembly of Gag Proteins in Vitro}

Recently, the possibility of establishing in vitro systems for the assembly of retroviral capsids has been explored. Gag proteins produced in bacteria have been successfully assembled into capsid-like structures. Fragments of either the RSV or HIV Gag precursor equivalent to the CA plus NC domains can be assembled into rod-shaped coils when combined with RNA (Campbell and Vogt, 1995). A larger fragment of RSV Gag comprising the entire precursor minus the proteinase domain can be similarly assembled in the presence of RNA, but in this case, forms spherical particles like those produced by proteinasedefective mutants of RSV (Campbell and Vogt, 1997). For either rods or spheres the assembly process appears to be independent of the RNA sequence since random $E$. coli RNA can serve as a suitable co-ssembly substrate. Studies of M-PMV gag expression in bacteria have demonstrated that capsids can assemble in $E$. coli. Capsid-like structures can be seen by thin-section electron microscopy within cellular inclusion bodies. Most importantly, capsid structures can be reassembled from these inclusion bodies after solubilization in urea (Klikova et al., 1995)

A second in vitro approach has been to produce Gag precursors in in vitro translation systems. Production of the Gag and Gag-Pro precursors of M-PMV in a reticulocyte system leads to the formation of immature capsid-like structures (Sakalian et al., 1996). In support of the authenticity of this system M-PMV mutant gag gene products which are defective for capsid assembly in vivo are also assembly incompetent in vitro. While the ability of HIV Gag to assemble into particles in a reticulocyte system remains controversial (Sakalian et al., 1996; Spearman and Ratner, 1996) efficient assembly of such particles was recently demonstrated in a wheat germ translation system (Lingappa et al., 1997). In addition, non-hydrolysable ATP analogs can block the formation of both HIV (Lingappa et al., 1997) and M-PMV (Sakalian and Hunter, 1997) particles in these in vitro systems, thus establishing a requirement for energy for the process of assembly itself. Furthermore, recent results with M-PMV have demonstrated that this in vitro assay system can distinguish true assembly mutants from intracellular transport mutants. The D- to C-type MA mutant, $\mathrm{R} 55 \mathrm{~W}$, can assemble in vitro demonstrating that it does not contain an assembly defect while p12 deletion mutants which fail to assemble in cells likewise fail to assemble in vitro demonstrating the defect is not merely one in transport (Sakalian and Hunter, 1997). The further development of these cell-free assembly systems will not only advance the molecular dissection of capsid assembly but, as has been demonstrated by the use of antiGag monoclonal antibodies, could facilitate the analysis of potential inhibitors of retrovirus capsid assembly (Sakalian et al., 1996).

\subsection{Incorporation of RNA}

During assembly, in a process that is not understood but for which no viral gene product other then Gag is necessary, two copies of the viral RNA genome are packaged (Berkowitz et al., 1996). The retrovirus RNA genome contains in the 5' untranslated region a sequence element that mediates packaging of viral RNA. In most retroviruses, this element is near to, or perhaps coincident with, the dimer linkage site, the sequence through which the two identical RNA subunits in each virion are held together. In many retroviruses, stemloop structures have been identified as important components the packaging element. 
What corresponding determinants in Gag recognize and bind to $\Psi$ RNA? Extensive studies of the NC protein, as a part of the Gag precursor, have established the importance of this domain in the packaging of genomic RNA (Berkowitz et al., 1996). Analysis of specific RNA packaging by a series of RSV Gag deletion mutants pointed to a possible role for sequences corresponding to the I assembly domain (Sakalian et al., 1994) leading again, as already mentioned, to the idea that RNA, whether viral or cellular, is required for assembly.

\subsection{Membrane Association and Extrusion}

Irrespective of the pathway followed by the Gag precursors, at a point either during assembly or following assembly in the cytoplasm, the nascent capsid must associate with the plasma membrane and induce the lipid bilayer to extrude; a process termed budding. It is clear that the viral glycoproteins are not necessary for this process to occur, since viruses lacking the env gene efficiently assemble and release membrane-enveloped virions.

It seems likely that during the process of membrane association and virus budding, conformational changes in the capsid precursors will occur. For the preassembled capsids of the type-D retroviruses, it is probable that the hydrophobic myristic acid modification of MA is buried inside the capsid structure during its transport through the hydrophilic environment of the cytoplasm. However, once the capsid has associated with the inner surface of the lipid bilayer, a conformational change which results in the exposure of this hydrophobic fatty acid on its surface might be expected to provide a more stable Gag-lipid interaction and could even provide the force for driving the budding process itself. A similar conformation-change driven process might also operate during C-type retroviral budding.

The final step in virus particle release requires the 'pinching-off' from the cell membrane of the immature particle - a process that involves a membrane fusion event. Although we now know that the $\mathrm{L}$ domain is necessary for this process the mechanism by which this occurs is a mystery. It is difficult to reconcile the positionally independent function of this domain with a hypothesis for a single cellular factor. However, it has been suggested recently that the L domain could constitute a binding site for "WW" proteins which contain a 38 amino acid sequence motif with two widely spaced tryptophan residues (Garnier et al., 1996). How such WW motif proteins, which include cell signaling molecules and cytoskeletal proteins, might function in releasing enveloped virions is unknown.

\section{ASSEMBLY OF ENVELOPE GLYCOPROTEINS}

While the capsid precursor proteins are transported through the cytoplasm by ill-defined pathways, the surface components of the virion, the envelope glycoproteins, are directed to the site of assembly through the relatively well characterized 'secretory pathway' of the cell (reviewed in Einfeld, 1996). The envelope glycoprotein complex of replication competent retroviruses is comprised of two polypeptides, an external, glycosylated, hydrophilic protein (SU) and a membrane-spanning protein (TM), that form a knob or knobbed spike on the surface of the virion. Both polypeptides are encoded by the env gene in the order - $\mathrm{NH}_{2}$--SU--TM--COOH - and are synthesized in the form of a glycosylated, polyprotein precursor that oligomerizes in the rough endoplasmic reticulum. This assembled complex is then transported to the Golgi where the attached oligosaccharides are processed 
and the precursor is proteolytically cleaved to its mature subunits. The glycoprotein oligomers must then be transported from the Golgi to the surface of the cell in order to participate in the assembly process. While the glycoproteins are not required for the assembly of enveloped virus particles, they do play a critical role in the virus replication cycle by recognizing and binding to specific receptors and by mediating the fusion of viral and cell membranes; virus particles lacking envelope glycoproteins are thus non-infectious.

What directs these nascent glycoproteins into budding virions? It has been generally accepted that they are preferentially incorporated into virus while the bulk of the host cell membrane proteins are excluded from the assembling particle. There is biochemical and genetic evidence for an interaction between the MA domain of capsid and the cytoplasmic domain of the TM protein (reviewed in Hunter, 1994). Mutations within both the MA protein and the TM protein coding domains of HIV-1 and M-PMV have been shown to reduce the efficiency of glycoprotein incorporation into virions indicating that interactions between these components of the virus might be important during the assembly process. Moreover, in polarized epithelial cells, the HIV glycoprotein can specifically target HIV capsid assembly and release to the basolateral plasma membrane. This data suggests that retroviral glycoproteins are positively selected from the mixture of molecules in the plasma membrane.

While such a positive selection model is attractive, it does not explain the facility with which foreign glycoproteins can be incorporated into retrovirus particles or the lack of effect deletion of the cytoplasmic domain of the TM protein can have on glycoprotein incorporation in some retroviral systems. In the case of RSV and SIV, truncation of the cytoplasmic domain does not block glycoprotein incorporation . Furthermore, a glycolipid anchored form of the HIV-1 glycoprotein complex was also efficiently incorporated into virus particles, suggesting that the protein anchor and cytoplasmic domain are not critical signals for incorporation (reviewed in Einfeld, 1996; Hunter, 1994).

The fact that a variety of non-retroviral glycoproteins can be assembled efficiently into retrovirus particles and in some viruses removal of the cytoplasmic domain does not prevent glycoprotein incorporation, is inconsistent with a specific requirement for strong capsid-glycoprotein interactions. How then might viral proteins be preferentially incorporated into virions? Given an intimate $\mathrm{MA} / \mathrm{membrane}$ association, it is possible that as virus assembly occurs, the associated areas of the plasma membrane are cleared of cellular glycoproteins that have connections with cytoplasmic counterparts (cytoskeletal elements or associated kinases for example). This active exclusion of cellular proteins might then allow mobile viral (and cellular) membrane proteins to freely diffuse into this region and be passively incorporated into virions. Such an active exclusion/passive inclusion mechanism would not preclude interactions between the capsid and glycoprotein, but neither would it require this interaction to take place for the glycoprotein complex to be incorporated into virus. Support for this hypothesis comes from more recent studies of HIV glycoprotein incorporation. In the context of mutations in the MA domain of Gag the envelope glycoprotein is excluded from virions; however, truncated Env species can be incorporated (Freed and Martin, 1995).

Indeed it may be possible to reconcile the apparent contradiction of a requirement for specific capsid/glycoprotein interactions during assembly of HIV and the 'passive incorporation' of foreign glycoproteins. Internalization signals have been found in the cytoplasmic domain of the TM proteins of HIV and SIV which can decrease the level of a glycoprotein on the cell surface (LaBranche et al., 1995). This same internalization signal, consisting of a membrane proximal tyrosine residue in the context of a "tight turn" motif has also been identified as the signal for basolateral membrane targeting of both the envelope glycopro- 
tein and virion budding (Lodge et al., 1997). The efficiency of glycoprotein incorporation could therefore reflect an equilibrium between competing interactions of viral glycoprotein endocytosis and viral glycoprotein/capsid association. Thus, mutations which interfere with the capsid/viral glycoprotein interaction reduce the efficiency with which glycoprotein is incorporated into virus due to internalization while mutations which remove endocytosis signals from the TM cytoplasmic domain result in higher levels of virion-associated glycoprotein. Recent results with HIV are consistent with this in that the Gag precursor can indeed suppress the endocytosis of the envelope glycoprotein while a mutant Gag that is unable to mediate glycoprotein incorporation cannot (Egan et al., 1997).

\section{VIRUS MATURATION}

The final stage in the assembly of an infectious retrovirus particle occurs following release of the immature virion and involves its conversion to a mature virus. The process of maturation requires proteolysis of the Gag and Gag-related precursors by the viral protease and a reorganization of the mature protein products and viral nucleic acids (reviewed in Vogt, 1996). During this process an electron dense core forms (Figure 1), the diploid viral genome forms a stable hydrogen-bonded dimer RNA structure and the virus becomes an infectious entity. What mechanism regulates the activation of the viral protease remains unknown. However, because the type-D viruses preassemble an immature capsid containing an inactive protease precursor, activation by concentrating the precursors in an assembling capsid is unlikely. It is possible that conformational changes in the capsid precursor during association with the membrane trigger protease activity. Mutations or inhibitors that block proteolysis prevent the formation of a mature virus and block infectivity (reviewed in Vogt, 1996). This process of maturation, which results in a relatively fragile, more easily disrupted structure, irreversibly commits the virus to the infection pathway and can be considered both the finale and the beginning of the virus life cycle.

\section{REFERENCES}

Berkowitz, R., Fisher, J., and Goff, S. P., 1996, RNA packaging, Curr. Top. Microbiol. Immunol. 214: $177-218$.

Bodem, J., Löchelt, M., Winkler, I., Flower, R. P., Delius, H., and Flügel, R. M., 1996, Characterization of the spliced pol transcript of feline foamy virus: the splice acceptor site of the pol transcript is located in gag of foamy viruses, J. Virol. 70: 9024-9027.

Campbell, S., and Vogt, V. M., 1995, Self assembly in vitro of purified CA-NC proteins from Rous sarcoma virus and human immunodeficiency virus type1, J. Virol. 69: 6487-6497.

Campbell, S., and Vogt, V. M., 1997, In vitro assembly of virus-like particles with Rous sarcoma virus gag deletion mutants: Identification of the p10 domain as a morphological determinant in the formation of spherical particles, J. Virol. 71: 4425-4435.

Conte, M. R., Klikova, M., Hunter, E., Ruml, T., and Matthews, S., 1997, The three-dimensional solution structure of the matrix protein from the type D retrovirus, the Mason-Pfizer Monkey virus, submitted.

Craven, R. C., and Parent, L. J., 1996, Dynamic interactions of the Gag polyprotein, Curr. Top. Microbiol. Immunol. 214: 65-94.

Egan, M. A., Carruth, L. M., Rowell, J. F., Yu, X., and Siliciano, R. F., 1997, Human immunodeficiency virus type 1 envelope protein endocytosis mediated by a highly conserved intrinsic internalization signal in the cytoplasmic domain of gp41 is suppressed in the presence of the Pr55gag precursor protein, J. Virol. 70: 6547-6556.

Einfeld, D., 1996, Matruation and assembly of retroviral glycoproteins, Curr. Top. Microbiol. Immunol. 214: 133-176. 
Eisenman, R. N., Vogt, V. M., and Diggelmann, H., 1974, Synthesis of avian RNA tumor virus structural proteins, Cold Spring Harbor Symp. Quant. Biol. 39: 1067-1075.

Freed, E. O., and Martin, M. A., 1995, Virion incorporation of envelope glycoproteis with long but not short cytoplasmic tails is blocked by specific, single amino acid substitutions in the human immunodeficiency virus type 1 matrix., J. Virol. 69: 1984-1989.

Garnier, L., Wills, J. W., Verderame, M. F., and Sudol, M., 1996, WW domains and retrovirus budding, Nature (London) 381: 744-745.

Gelderblom, H. R., 1991, Assembly and morphology of HIV: potential effect of structure on viral function, AIDS 5: 617-638.

Hunter, E., 1994, Macromolecular interactions in the assembly of HIV and other retroviruses, Sem. in Virology 5 : $71-83$.

Jacks, T., 1990, Translational suppression in gene expression in retroviruses and retrotransposons, Curr. Top. Microbiol. Immunol. 157: 93-124.

Katz, R. A., and Jentoft, J. E, 1989, What is the role of the cys-his motif in retroviral nucleocapsid (NC) proteins?, BioEssays 11: 176-181.

Klikova, M., Rhee, S. S., Hunter, E., and Ruml, T., 1995, Efficient in vivo and in vitro assembly of retroviral capsids from Gag precursor proteins expressed in bacteria, J. Virol. 69: 1093-1098.

Kräusslich, H.-G., and Welker, R., 1996, Intracellular transport of retroviral capsid components, Curr. Top. Microbiol. Immunol. 214: 25-64.

LaBranche, C. C., Sauter, M. M., Haggarty, B. S., Vance, P. J., Romano, J., Hart, T. K., Bugelski, P. J., Marsh, M., and Hoxie, J. A., 1995, A single amino acid change in the cytoplasmic domain of SIVmac transmembrane molecule increases envelope glycoprotein expression on infected cells, J. Virol. 69: 5217-5227.

Leis, J., Baltimore, D., Bishop, J. M., Coffin, J., Fleissner, E., Goff, S. P., Oroszlan, S., Robinson, H., Skalka, A. M., Temin, H. M., and Vogt, V., 1988, Standardized and simplified nomenclature for proteins common to all retroviruses, $J$. Virol. 62: 1808-1809.

Lingappa, J. R., Hill, R. L., Wong, M. L., and Hegde, R. S., 1997, A multistep, ATP-dependent pathway for assembly of human immunodeficiency virus capsids in a cell-free system, J. Cell Biol. 136: 567-581.

Linial, M. L., and Miller, A. D., 1990, Retroviral RNA packaging: Sequence requirements and implications, Curr. Top. Microbiol. Immunol. 157: 125-152.

Lodge, R., Lalonde, J.-P., Lemay, G., and Cohen, E. A., 1997, The membrane-proximal intracytoplasmic tyrosine residue of HIV-1 envelope glycoprotein is critical for basolateral targeting of viral budding in MDCK cells, EMBO J. 16: 695-705.

Matthews, S., Mikhailov, M., Burny, A., and Roy, P., 1996, The solution structure of the bovine leukaemia virus matrix protein and similarity with lentiviral matrix proteins, EMBO J. 15: 3267-3274.

Nermut, M. V., and Hockley, D. J., 1996, Comparative morphology and structural classification of retroviruses, Curr. Top. Microbiol. Immunol. 214: 1-24.

Sakalian, M., and Hunter, E., 1997, Unpublished results.

Sakalian, M., Parker, S. D., Weldon, R. A., Jr., and Hunter, E., 1996, Synthesis and assembly of retrovirus gag precursors into immature capsids in vitro, J. Virol. 70: 3706-3715.

Sakalian, M., Wills, J. W., and Vogt, V. M., 1994, Efficiency and selectivity of RNA packaging by Rous sarcoma virus Gag deletion mutants, J. Virol. 68: 5969-5981.

Sommerfelt, M. A., Rhee, S. S., and Hunter, E., 1992, Importance of the p12 protein in Mason-Pfizer monkey virus assembly and infectivity, J. Virol. 66: 7005-7011.

Spearman, P., and Ratner, L., 1996, Human immunodeficiency virus type 1 capsid formation in reticulocyte lyates, J. Virol. 70: 8187-8194.

Vogt, V. M., 1996, Proteolytic processing and particle maturation, Curr. Top. Microbiol. Immunol. 214: 95-131.

Weber, I., 1990, Comparison of the crystal structures and intersubunit interactions of human immunodeficiency and Rous sarcoma virus proteases, J. Biol. Chem. 265: 10492-10496.

Weldon, R. A. J., and Hunter, E., 1997, Unpublished results.

Yasuda, J., and Hunter, E., 1997, Unpublished results. 